\title{
Numerical Simulation of Chromatic Dispersion and Fiber Attenuation in a Single-Mode Optical Fiber System
}

\author{
S. H. Lawan ${ }^{1}$, M. Ajiya ${ }^{2}$ and D. S. Shu'aibu ${ }^{3}$ \\ ${ }^{1,2,3}$ (Department of Electrical Engineering Bayero University, Kano-Nigeria)
}

\begin{abstract}
Chromatic dispersion and fiber attenuation pose a great problem in the detection of optical signals. Dispersion causes pulse broadening which limits the information carrying capacity of the fiber while attenuation limits the maximum transmission distance along the fiber. An approximate Gaussian pulse propagation model is obtained from the solution of Nonlinear Schrödinger Equation to represent the effects of chromatic dispersion and attenuation using Split-Step Fourier Method. It was found that pulse broadening and intensity loss in the optical signal is increasing proportionately with the propagation length of the fiber and this is what contributes to the causes of detection errors at the receiver.
\end{abstract}

Keywords - Chromatic dispersion, fiber attenuation, intensity loss, optical signals, pulse broadening

\section{Introduction}

Optical fibers are becoming dominant transmission media in long distance transmission, optical fiber transmission is used from a short distance below $1 \mathrm{ft}$ up to transoceanic distances in undersea cable. In fact all currently future plans in transoceanic cables are fiber optic based. Advantages of fiber optic as a medium of transmission are many from unlimited bandwidth, immunity from both radio frequency interference (RFI) and electromagnetic interference (EMI) to excellent attenuation properties, when compared with other transmission media like coaxial cable [1].

Attenuation is the loss of optical power as light travels along the fiber [2,3]. Signal attenuation is defined as the ratio of optical input power $\left(P_{i n}\right)$ to the optical output power $\left(P_{\text {out }}\right)$ [3]. Optical input power is the power injected into the fiber from an optical source. Optical output power is the power received at the fiber end or optical detector.

An intrinsic limit to the information carrying capacity of optical fibers is set by the wavelength dependence of the propagation constant, resulting in chromatic dispersion and in consequent bandwidth penalty[4]. Hence, the need of an efficient measurement technique to evaluate chromatic dispersion properties of optical fibers in an accurate and possibly simple way [4]. Chromatic dispersion in an optical fiber is the limiting factor in achieving very high bandwidth communication systems [5]. Recent progress in optical fiber amplifier technology makes fiber dispersion the ultimate limiting factor for high-speed long-distance optical fiber transmission [6]. Dispersion is the spreading out of a light pulse in time as it propagates down the fiber [3]. This is the dispersion spread that results in system bandwidth reduction or the fiber information-carrying capacity, it limits how fast information is transferred [7]. The chromatic dispersion of optical fiber is critical to the design and construction of long-haul and high-speed optical communication systems and to the manufacture of optical fiber [8]. it is important to reduce the accumulated chromatic dispersion after long distance transmission [9]. Dispersion in optical fiber includes modal dispersion and chromatic dispersion, chromatic dispersion consist of material dispersion and waveguide dispersion [10].

Material dispersion is the result of the finite linewidth of the light source and the dependence of refractive index of the material on wavelength [11, 12]. Material dispersion is a type of chromatic dispersion. Waveguide dispersion is only important in single mode fibers[3]. It is caused by the fact that some light travels in the fiber cladding compared to most light travels in the fiber core [13]. Since fiber cladding has lower refractive index than fiber core, light ray that travels in the cladding travels faster than that in the core [13].

\section{Methodology}

The pulse envelope in time $t$ at the spatial position $z$, propagating from transmitting to the receiving end of an optical fiber communication system is $A(z, t)$. Pulse propagation in an optical fiber communication system is described by the non-linear Schrödinger equation (NLSE) [2].

Where

$$
\frac{\partial A}{\partial z}+\frac{\beta_{1} \partial A}{\partial t}-\frac{i}{2} \frac{\beta_{2} \partial^{2} A}{\partial t^{2}}+\frac{\alpha}{2} A=-i \alpha_{o}|A|^{2} A
$$

$A$ is the pulse envelope in spatial position $z$ and in time $t$.

$\beta_{1}$ in the first order dispersion parameter causes pulse delay due to polarization mode dispersion.

$\beta_{2}$ is the second order dispersion parameter causes pulse broadening due to chromatic dispersion. 
$\alpha$ is the attenuation coefficient of the fiber and

$\alpha_{o}$ is the non-linearity coefficient of the fiber and it is a function of the light strength.

$i$ is a complex vector notation

The solution of Equation (1) is obtainable using split-step Fourier method.

Considering the effect of chromatic dispersion and fiber attenuation only and that the pulse position is at zero center-frequency, the solution of Equation (1) yields.

$$
A(L, \omega)=\exp \left[\left(\frac{i \beta_{2}}{2} \omega^{2}-\frac{\alpha}{2}\right) L\right] A(0, \omega)
$$

It is clear from the above equation that the signal amplitude decreases as it is propagating along the length of optical fiber this causes power loss and CD affects the phase of the signal spectrum without changing the spectral power distribution and thereby causing the propagated pulse to be broadened.

The following equation defines signal attenuation as a unit of length [2]

$$
\alpha_{d B}=-\frac{10}{L} \log _{10}\left(\frac{P_{\text {out }}}{P_{\text {in }}}\right)
$$

Signal attenuation is a $\log$ relationship. Length $(L)$ is expressed in kilometers. Therefore, the unit of attenuation is decibels/kilometer $(\mathrm{dB} / \mathrm{km})$. The attenuation constant $\alpha$ is in per kilometer and mostly expressed in $\mathrm{dB}$ as $\alpha_{d B}$ [2]

$$
\alpha_{d B} \approx 4.343 \alpha
$$

There are different values of attenuation depending on the optical frequency range, here the window with least attenuation of 0.2 to $0.25 \mathrm{~dB} / \mathrm{km}$ which corresponds to wavelength of around $1550 \mathrm{~nm}$ is used. $\beta_{2}$ is called the group velocity dispersion (GVD) or simply dispersion, the time delay between two different spectral components separated by a certain frequency interval is determined using the dispersion coefficient $D$ given by [2]

$$
\begin{aligned}
& D=-\frac{2 \pi c}{\lambda^{2}} \beta_{2} \\
& \text { or } \beta_{2}=-\frac{\lambda^{2}}{2 \pi c} D
\end{aligned}
$$

Where $c$ is the speed of light and $\lambda=2 \pi c / \omega$ is the carrier wavelength, the time delay between two different spectral component separated by a certain wavelength interval in determined by the dispersion coefficient $D$ in $\mathrm{ps} / \mathrm{nm}-\mathrm{km}$. The propagation distance after which a Gaussian pulse is broadened by $40 \%$ is termed the dispersion length and is given by [2]

$$
L_{D}=-\frac{t_{o}{ }^{2}}{\left|\beta_{2}\right|}
$$

where $t_{\mathrm{o}}$ is the pulse full-width at half-maximum (FWHM)

The input is represented by a Gaussian pulse and it is defined as [2]

$$
A(t)=\exp \left(-\frac{1}{2}\left(t / t_{o}\right)^{2}\right)
$$

In general, a simulation is a computer model of a part of a real-world system. Here the simulation is a computer model of a single mode optical fiber link system, includes attenuation function and chromatic dispersion function. The attenuation is added in either time or frequency domain but the chromatic dispersion effect must be added in frequency domain. The transformation of this signal from time to frequency to time is achieved using Fast Fourier Transform (FFT) and Inverse Fast Fourier Transform (IFFT) [7].

The input signals are the signals that will be sent through the fiber link, or the output signals of the light sources. These signals are converted in to a shape that is suitable for computer simulation. So, the input signal must first be represented in the form of a numeric array. The array contains samples of the amplitude profile at a number of equally spaced points. The sampling resolution must be fine enough to resolve all spatial features of the amplitude profile, at the same time it must sparse enough to allow reasonable processing speed on a computer. The type of pulse that used in the simulation is Gaussian pulse. The Gaussian pulse is used because the optical sources have a distribution of power with wavelength that is approximately Gaussian distribution in form [7]. 


\section{Results And Discussion}

Gaussian pulse was taken as an input signal and the effects of chromatic dispersion and attenuation are studied. The effect of attenuation is shown in Figure 1. and that of chromatic dispersion is shown in Figure 3. for distances up to $200 \mathrm{~km}$ at $50 \mathrm{~km}$ interval. Linear relation of Pulse broadening due chromatic dispersion in $\mathrm{ps} / \mathrm{nm}$ $\mathrm{km}$ and fiber attenuation in $\mathrm{dB}$ with transmission length of fiber in $\mathrm{km}$ are obtained as shown below in Figure 2. And Figure 4. Respectively.

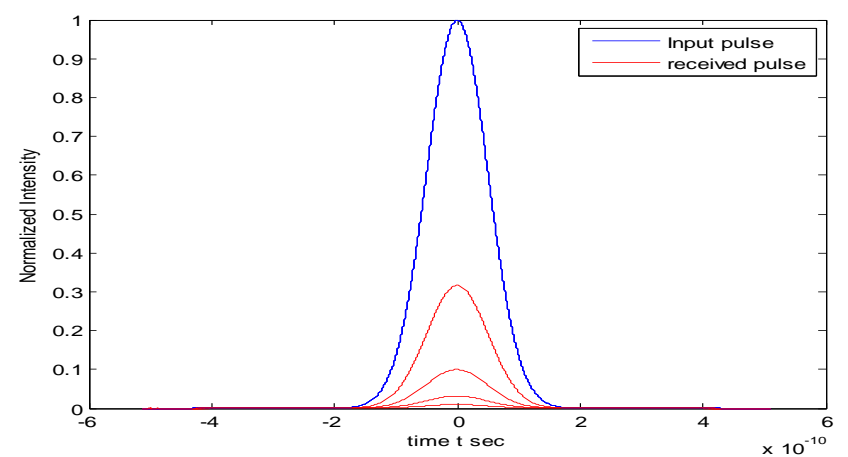

Figure 1. Attenuation effects at 50,100,150 and 200km distance

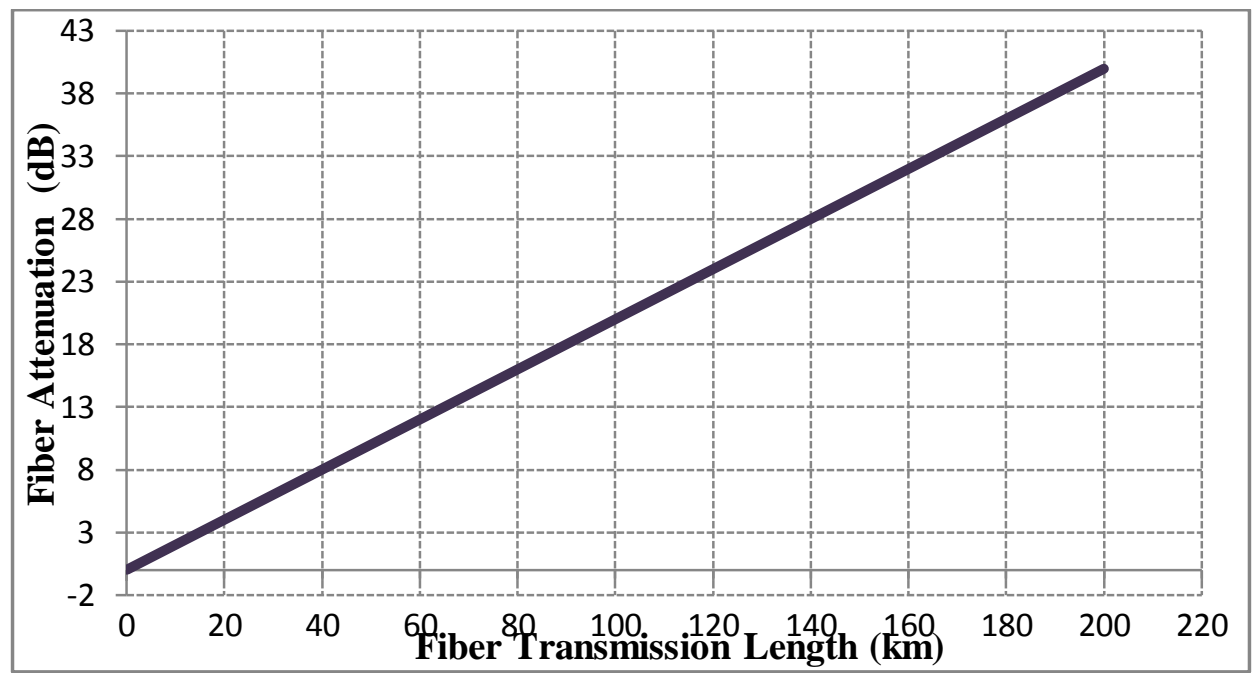

Figure 2. Fiber Attenuation

Signal intensity loss due to fiber attenuation increases with propagation distance as shown in Figure 2. fiber attenuation effect on the input pulse is shown in purple with a slope of $0.2 \mathrm{~dB} / \mathrm{km}$.

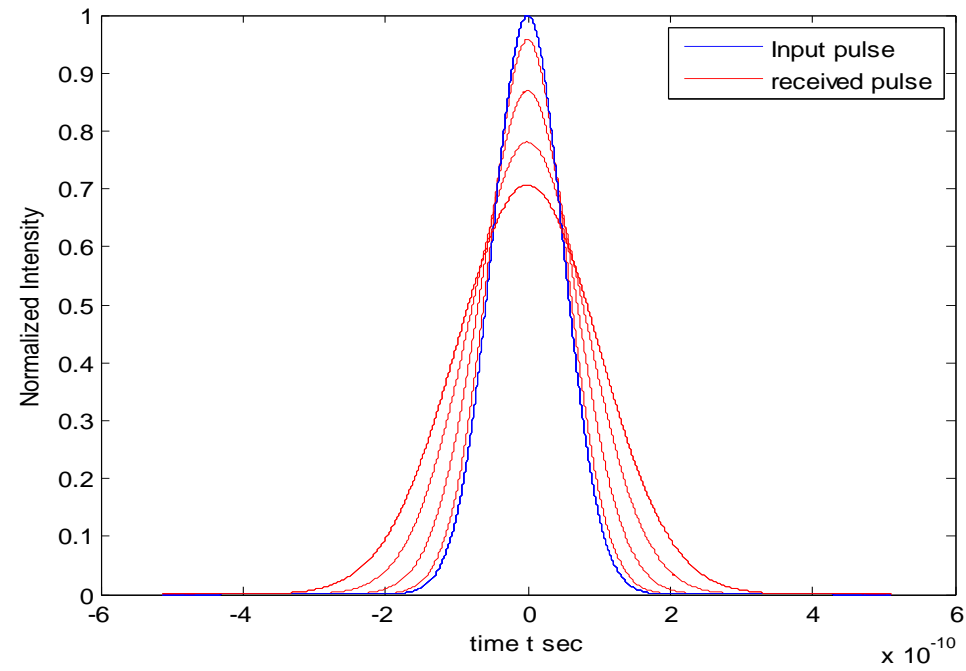

Figure 3. Dispersion effects at 50,100, 150 and 200km distance 


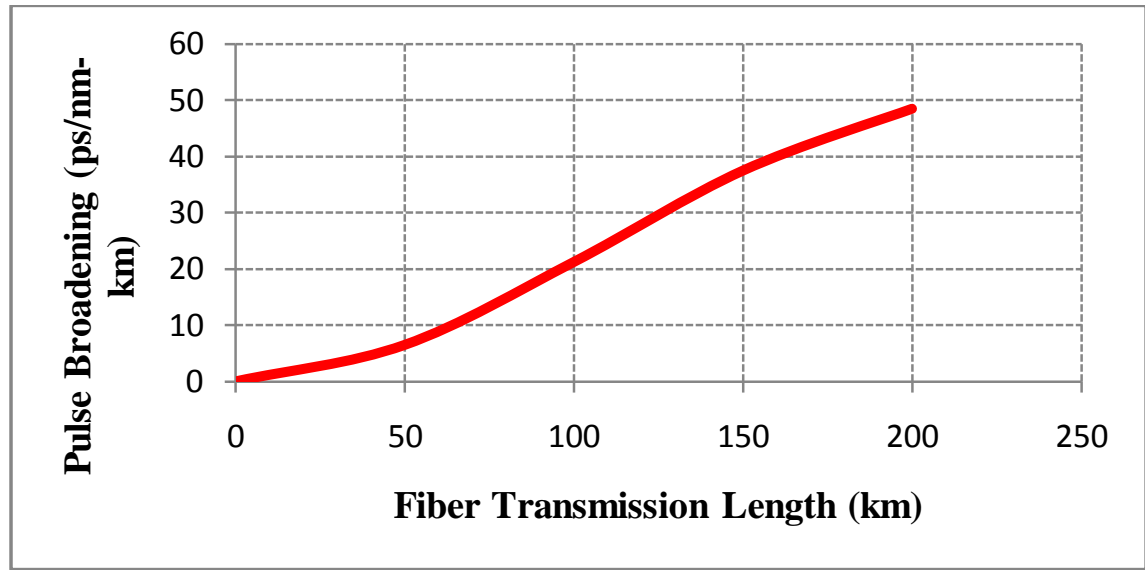

Figure 4. Pulse Broadening due to Chromatic Dispersion

Pulse broadening due to chromatic dispersion in the optical signal is increasing proportionately with the propagation length of the fiber as shown in Figure 4.

\section{Conclusion}

The results indicate that the linear effects of chromatic dispersion and attenuation increase with increasing the distance along the fiber optic length. As the input signal propagates degradation occur due to these effects and eventually lead to detection problem. Therefore at certain distances there is need for repeaters and dispersion management system to overcome such signal distortions.

\section{References}

[1] K. Thyagarajan and A. Ghatak, Fiber Optic Essentials. New York: John Wiley \& Sons. Inc., 2007.

[2] G. P. Agrawal, Nonlinear Fiber Optics, 3rd ed. UK: Academic Press. A Harcourt Sci. and Tec. Co., 2001

[3] G. P. Agrawal, Fiber-optic Communication Systems. New York: John Wiley \& Sons. Inc., 2002.

[4] B. Costa, D. Mazzoni, M. Puleo, and E. Vezzoni, "Phase shift technique for the measurement of chromatic dispersion in optical fibers using LED's," IEEE Journal of Quantum Electronics, vol. 18, pp. 1509-1515, 1982.

[5] P. Merritt, R. P. Tatam, and D. A. Jackson, "Interferometric chromatic dispersion measurements on short lengths of monomode optical fiber," Journal of Lightwave Technology, vol. 7, pp. 703-716, 1989.

[6] A. E. N. A. Mohammed, "New technique of chromatic dispersion management in optical single-mode binary glass fiber," 2004, pp. D9-1-12.

[7] S. H. S. Al-Bazzaz, "Simulation of Single mode fiber optics and optical communication Components Using VC++," International Journal of Computer Science and Network Security, vol. 8, pp. 300-308, 2008.

[8] K. S. Jeon, H. J. Kim, D. S. Kang, and J. K. Pan, "Optical fiber chromatic dispersion measurement using bidirectional modulation of an optical intensity modulator," Photonics Technology Letters, IEEE, vol. 14, pp. 1145-1147, 2002.

[9] H. Taga, S. Yamamoto, N. Edagawa, Y. Yoshida, S. Akiba, and H. Wakabayashi, "Fiber chromatic dispersion equalization at the receiving terminal of IM-DD ultra-long distance optical communication systems," Journal of Lightwave Technology, vol. 12, pp. 1042-1046, 1994.

[10] G. P. Agrawal, Fiber-Optic Communication Systems, 3rd ed. NJ: Wiley, Hoboken, 2002

[11] C. R. Doerr and K. Okamoto, "Advances in silica planar lightwave circuits," Journal of Lightwave Technology, vol. 24, pp. 47634789, 2006

[12] K. Okamoto, Fundamentals of optical waveguides: Academic press, 2006.

[13] H. J. R. Dutton and I. B. M. Corporation, Understanding optical communications: Prentice Hall PTR, 1998. 